Discussion Paper Series A No.523

\title{
Strong Subjectivism in the Marxian Theory of Exploitation: A Critique
}

\author{
Roberto Veneziani \\ (Department of Economics, Queen Mary University of London) \\ and \\ Naoki Yoshihara \\ (Institute of Economic Research, Hitotsubashi University)
}

November, 2009

Institute of Economic Research

Hitotsubashi University

Kunitachi, Tokyo, 186-8603 Japan 


\title{
Strong Subjectivism in the Marxian Theory of Exploitation: A Critique*
}

\author{
Roberto Veneziani ${ }^{\dagger}$ and Naoki Yoshihara ${ }^{\ddagger}$
}

November 2009

\begin{abstract}
This paper critically analyses the strongly subjectivist approach to exploitation theory recently proposed by Matsuo ([7]), in the context of general convex economies with heterogeneous agents. It is proved that the Fundamental Marxian Theorem is not preserved in his subjectivist approach, contrary to Matsuo's claims, and that no meaningful subjectivist exploitation index can be constructed. It is argued that a minimal objectivism is necessary in exploitation theory, whereby subjective preferences do not play a direct, definitional role. An alternative objectivist approach is briefly analysed, which is related to the 'New Interpretation' ([1]; [3]). It is argued that it captures the core intuitions of exploitation theory and it provides appropriate indices of individual and aggregate exploitation. Further, it is shown that it preserves the FMT in general economies.
\end{abstract}

JEL Classification Numbers: D31; D46; D63; E11; B51.

Keywords: Exploitation, subjective preferences, Fundamental Marxian Theorem, general convex cone economies.

${ }^{*}$ We are indebted to participants in the Hitotsubashi University workshop on Exploitation Theory and to two anonymous referees for many insightful comments and suggestions. This research started when Roberto Veneziani was visiting Hitotsubashi University. Their generous hospitality and financial support is gratefully acknowledged. The usual disclaimer applies.

${ }^{\dagger}$ Department of Economics, Queen Mary University of London, Mile End Road, London E1 4NS, UK. E-mail: r.veneziani@qmul.ac.uk

†The Institute of Economic Research, Hitotsubashi University, Naka 2-1, Kunitachi, Tokyo 186-0004, Japan. Phone: (81)-42-580-8354, Fax: (81)-42-580-8333. e-mail: yosihara@ier.hit-u.ac.jp 


\section{Introduction}

In the Marxian theory of exploitation, workers are said to be exploited if the labour they expend is higher than the amount of labour contained in some relevant bundle of wage goods, which measures the value of labour power. This definition is seemingly intuitive, but even in stylised two-class societies, it has proved surprisingly difficult to provide a fully satisfactory general theory of exploitation. First, outside the standard Leontief economy, the definition of exploitation is ambiguous, for the appropriate definition of the value of labour power is not obvious, and indeed a number of approaches have been proposed (see [19]; [21]). In turn, this implies that the definition of the exploitation index, measuring the amount of exploitation in the economy, is controversial. This is a central issue because a theory of exploitation should be able to compare different societies in terms of their exploitation levels, and to analyse the evolution of an economy, and its exploitation structure, over time. Second, outside the Leontief setting, the core insights of exploitation theory do not necessarily hold. For example, in more general economies, a number of counterexamples to the so-called Fundamental Marxian Theorem (FMT) have been produced. In standard approaches, this is also an important issue because the FMT proves that exploitation is synonymous with positive profits. The relevance of the FMT is such that although it is proved as a result, its epistemological status is that of a postulate: the appropriate definition of exploitation, and of an exploitation index, is considered to be one which preserves the FMT. A number of definitions of exploitation, and exploitation indices, have been proposed precisely in an attempt to generalise the FMT to economies with joint production, heterogenous labour, and so on. (see, e.g., [10]; [6]; [12]. For recent debates, see [14]; [19].)

The main approaches in the literature define exploitation in relation to the objective features of an economy (including data on production, consumption, labour supply, etc.), with no direct reference to individual attitudes, beliefs, and preferences. In a recent article, Matsuo [7] has proposed an original theory of exploitation, in which agents' preferences play a direct, definitional role: workers are exploited if and only if there is a bundle of goods that they weakly prefer to the wage goods they receive and that can be produced with less labour than they have expended. This approach can be defined as strongly subjectivist in order to distinguish it from other approaches in which preferences play an indirect role, e.g. via their influence on individual consumption and labour decisions. According to Matsuo, his 
definition of exploitation is superior to the alternatives because it avoids the standard counterexamples to the FMT (e.g. [12]; [13]).

This paper critically analyses Matsuo's subjectivist approach, focusing on the appropriate definition of an exploitation index and on the FMT, in general convex economies with heterogeneous agents. Although some key results of the subjectivist approach can be generalised, it is shown that, under different concepts of equilibrium, the FMT does not hold: contrary to Matsuo's claims, his subjectivist approach does not solve the problems of traditional theories of exploitation. Further, due to the definitional role of preferences, there is an inherent deep indeterminacy in his subjectivist measure of exploitation, such that no theoretically robust and empirically meaningful index can be constructed. It is argued that a minimal objectivism is necessary in exploitation theory and Matsuo's approach is contrasted with an objectivist definition of exploitation, which is conceptually related to the so-called 'New Interpretation' ([1]; [2]; [3]). It is shown that the latter approach is preferable according to both criteria considered in this paper: it provides a well-defined individual and aggregate exploitation index and it preserves the FMT in general convex economies with heterogeneous agents.

It is important to note at the outset that exploitation theory is not just about constructing an index of exploitation and proving the FMT, and an exhaustive analysis of exploitation in advanced capitalist economies cannot be limited to simple two-class models. Two points should be made here to motivate the focus of this paper, and the models analysed. First, the theoretical starting point - and the main object of critical analysis - is Matsuo's [7] innovative contribution, which raises the interesting issue of whether, and how, subjective preferences should count in the definition of exploitation. Therefore, his model is significantly generalised by allowing for heterogeneous preferences and consumption bundles, and for a convex cone technology, but some of the simplifying assumptions - such as the stark two-class structure are retained, for analytical and expositional purposes. Second, the construction of the aggregate exploitation index and the relation between profits and exploitation do not exhaust the discussion of the normative and positive relevance of exploitation, but they are arguably crucial and do play a prominent role in the literature, including in a number of recent articles appeared on this journal (e.g., [15]; [4]; [5]; [9]). The general convex economies considered in this paper are appropriate to analyse both issues and the simplifying assumptions made are standard in the literature. It is worth noting, though, that the 'New Interpretation' provides a general theoretical framework, which 
can deal with many unresolved issues in exploitation theory, in rather general economies. Several extensions of our analysis are briefly discussed in Section 6 below.

\section{The General Model}

In this section, a general model for the analysis of exploitation is provided along the lines of Roemer ([13], [14]), which allows for a general convex cone technology, rather than the standard von Neumann framework often used in exploitation theory. This is not just for the sake of formalism: the differences between alternative approaches to exploitation and the anomalies in the relation between profits and exploitation become relevant when the linear production model is abandoned.

\subsection{Production}

In the economy there are $n$ produced commodities and one non-produced good, namely labour. Let $\mathbf{0} \in \mathbb{R}^{n}$ be such that $\mathbf{0}=(0, \ldots, 0)$. Let $P$ be the production set: $P$ has elements of the form $\alpha=\left(-\alpha_{0},-\underline{\alpha}, \bar{\alpha}\right)$ where $\alpha_{0} \in \mathbb{R}_{+}, \underline{\alpha} \in \mathbb{R}_{+}^{n}$, and $\bar{\alpha} \in \mathbb{R}_{+}^{n}$. Thus, elements of $P$ are vectors in $\mathbb{R}^{2 n+1}$. The first component, $-\alpha_{0}$, is the direct labour input of the process $\alpha$; the next $n$ components, $-\underline{\alpha}$, are the inputs of goods used in the process; and the last $n$ components, $\bar{\alpha}$, are the outputs of the $n$ goods from the process. The net output vector arising from $\alpha$ is denoted as $\widehat{\alpha} \equiv \bar{\alpha}-\underline{\alpha}$. The set $P$ is assumed to be a closed convex cone containing the origin in $\mathbb{R}^{\frac{\alpha}{2 n}+1}$. Moreover, it is assumed that: ${ }^{1}$

Assumption (A1). $\forall \alpha \in P$ s.t. $\alpha_{0} \geq 0$ and $\underline{\alpha} \geqq \mathbf{0},\left[\bar{\alpha} \geq \mathbf{0} \Rightarrow \alpha_{0}>0\right]$;

Assumption (A2). $\forall c \in \mathbb{R}_{+}^{n}, \exists \alpha \in P$ s.t. $\widehat{\alpha} \geqq c$;

Assumption (A3). $\forall \alpha \in P, \forall\left(\underline{\alpha}^{\prime}, \bar{\alpha}^{\prime}\right) \in \mathbb{R}_{+}^{n} \times \mathbb{R}_{+}^{n}$,

$$
\left[\left(-\underline{\alpha}^{\prime}, \bar{\alpha}^{\prime}\right) \leqq(-\underline{\alpha}, \bar{\alpha}) \Rightarrow\left(-\alpha_{0},-\underline{\alpha}^{\prime}, \bar{\alpha}^{\prime}\right) \in P\right] .
$$

\footnotetext{
${ }^{1}$ For all vectors $x=\left(x_{1}, \ldots, x_{n}\right)$ and $y=\left(y_{1}, \ldots, y_{n}\right) \in \mathbb{R}^{n}, x \geqq y$ if and only if $x_{i} \geqq y_{i}$ $(i=1, \ldots, n) ; x \geq y$ if and only if $x \geqq y$ and $x \neq y ; x>y$ if and only if $x_{i}>y_{i}$ $(i=1, \ldots, n)$.
} 
A1 implies that labour is indispensable to produce any non-negative output vector; A2 states that any non-negative commodity vector is producible as a net output; and A3 is a free disposal condition for the production possibility set, which states that, given any feasible production process $\alpha$, any vector producing (weakly) less net output than $\alpha$ is also feasible using the same amount of labour as $\alpha$ itself.

Given $P$, the following notation is used:

$$
\begin{aligned}
P\left(\alpha_{0}=l\right) & \equiv\left\{\left(-\alpha_{0},-\underline{\alpha}, \bar{\alpha}\right) \in P \mid \alpha_{0}=l\right\}, \\
\widehat{P}\left(\alpha_{0}=l\right) & \equiv\left\{\widehat{\alpha} \in \mathbb{R}^{n} \mid \exists \alpha=(-l,-\underline{\alpha}, \bar{\alpha}) \in P \text { s.t. } \bar{\alpha}-\underline{\alpha} \geqq \widehat{\alpha}\right\}, \\
S \widehat{P}\left(\alpha_{0}=l\right) & \equiv\left\{\widehat{\alpha} \in \widehat{P}\left(\alpha_{0}=l\right) \mid \nexists \widehat{\alpha}^{\prime} \in \widehat{P}\left(\alpha_{0}=l\right) \text { s.t. } \widehat{\alpha}^{\prime} \geq \widehat{\alpha}\right\},
\end{aligned}
$$

where $P\left(\alpha_{0}=l\right)$ is the set of production vectors which use $l$ units of labour as an input, $\widehat{P}\left(\alpha_{0}=l\right)$ is the corresponding set of net outputs, and $S \widehat{P}\left(\alpha_{0}=l\right)$ is the set of net outputs that can be produced efficiently using exactly $l$ units of labour. Further, for any set $S \subseteq \mathbb{R}^{n}$, the set $\partial S \equiv\left\{x \in S \mid \nexists x^{\prime} \in S\right.$ s.t. $\left.x^{\prime}>x\right\}$ is the frontier of $S$ and $\stackrel{\circ}{S} \equiv S \backslash \partial S$ is its interior.

The von Neumann model with joint production (analysed, among the others, by Matsuo [7]) is a special case of the convex cone technology. Let $A$ be an $n \times m$ non-negative matrix with input coefficients $a_{i j} \geqq 0$ for any $i=1, \ldots, n, j=1, \ldots, m$, and $B$ be an $n \times m$ non-negative matrix with output coefficients $b_{i j} \geqq 0$ for any $i=1, \ldots, n, j=1, \ldots, m$. Moreover, let $L$ be a positive $1 \times m$ vector with labour input coefficients $L_{j}>0$ for any $j=1, \ldots, m$. To be precise, the von Neumann economy is a particular type of $P$, denoted as $P_{(A, B, L)}$, which can be described as follows

$P_{(A, B, L)} \equiv\left\{\left(-\alpha_{0},-\underline{\alpha}, \bar{\alpha}\right) \in \mathbb{R}_{-} \times \mathbb{R}_{-}^{n} \times \mathbb{R}_{+}^{n} \mid \exists x \in \mathbb{R}_{+}^{m}: \alpha_{0}=L x \&(-\underline{\alpha}, \bar{\alpha}) \leqq(-A x, B x)\right\}$.

This $P_{(A, B, L)}$ is a closed convex cone in $\mathbb{R}_{-} \times \mathbb{R}_{-}^{n} \times \mathbb{R}_{+}^{n}$ with $\mathbf{0} \in P_{(A, B, L)}$. Moreover, $P_{(A, B, L)}$ is easily shown to satisfy Assumptions 1-3.

\subsection{Agents}

In the standard two-class model used to analyse the FMT, the economy consists of a set $K$ of capitalists and of a set $W$ of workers. The set of agents $N$, with generic element $\nu$, is therefore given by $N=K \cup W$. To be specific, let $\omega^{\nu} \in \mathbb{R}_{+}^{n}$ denote the vector of initial productive endowments of agent $\nu \in N$ : the working class $W$ is the set of agents with no initial endowments, 
while the capitalist class $K$ is the set of agents endowed with some nonnegative and non-zero amount of inputs. Thus, $W=\left\{\nu \in N \mid \omega^{\nu}=\mathbf{0}\right\}$ and $K=\left\{\nu \in N \mid \omega^{\nu} \geq \mathbf{0}\right\}^{2}$

Each capitalist can operate any activity of the technology $P$ and is assumed to maximise profits. For the sake of simplicity, capitalists are also assumed to save all revenues, which are invested in the next production period, and to supply no labour (e.g., they can be assumed to derive infinite disutility from labour). Each worker is endowed with one unit of labour, which is assumed to be homogenous - there is no skill heterogeneity among workers. ${ }^{3}$ Let $b^{\nu}$ denote the consumption bundle of worker $\nu$ and let $l^{\nu}$ denote the labour performed by $\nu$. In what follows, we consider both economies with $b^{\nu}=b$ for all $\nu \in W$ and economies in which workers choose different bundles. In order to focus on the essential structure of Matsuo's approach, we assume that $l^{\nu}=l$, all $\nu \in W$.

In Matsuo's [7] subjectivist framework, the definition of exploitation requires the specification of the agents' (more precisely, the workers') utility functions. Thus, for every $\nu \in W, u^{\nu}: \mathbb{R}_{+}^{n+1} \rightarrow \mathbb{R}$ is the utility function representing $\nu$ 's preference over consumption and leisure. Given $c \in \mathbb{R}_{+}^{n}$ and $u^{\nu} \in \mathcal{U}$, let the upper contour set of $u^{\nu}$ at $c$ be given by $U\left(c ; u^{\nu}\right) \equiv\left\{c^{\prime} \in \mathbb{R}_{+}^{n} \mid u^{\nu}\left(c^{\prime}\right)>u^{\nu}(c)\right\}$. A convex cone economy is given by a list $E=\left\langle K, W ;\left(u^{\nu}\right)_{\nu \in W} ; P ;\left(\omega^{\nu}\right)_{\nu \in K}\right\rangle$, and the set of all such economies is denoted by $\mathcal{E}$. Finally, given a market economy, any price system is denoted by $p \in \mathbb{R}_{+}^{n}$, which gives one price for each of the $n$ commodities. Moreover, the nominal wage rate is assumed to be positive and equal to unity.

\footnotetext{
${ }^{2}$ In principle, one might argue that the appropriate definition of workers and capitalists relates to their financial wealth, rather than their vector of endowments. If this view is adopted, then $W=\left\{\nu \in N \mid p \omega^{\nu}=0\right\}$ and $K=\left\{\nu \in N \mid p \omega^{\nu}>0\right\}$. This distinction is relevant only if $p \ngtr \mathbf{0}$ and it does not make any significant difference for the results of this paper.

${ }^{3}$ The presence of heterogeneous labour does raise important issues in exploitation theory, including on the relation between exploitation and profits (for a discussion, see, e.g., [6]). Yet, this issue is not relevant in the comparison between objectivist and subjectivist approaches, which is the central theme of this paper. In his subjectivist approach to exploitation, [7] also assumes homogeneous labour (see Assumption 3).
} 


\section{A Strongly Subjectivist Approach to Ex- ploitation}

Matsuo [7] only considers the rather special case of von Neumann economies with identical workers. Given the emphasis on individual preferences, the representative agent setting seems unduly restrictive and in this section his analysis is generalised to convex economies with heterogeneous agents.

In Matsuo's framework, workers' preferences do not play a merely subsidiary, or indirect role (e.g., in determining consumption and leisure choices): utility functions play a direct, definitional role. Consider first the definition of labour values and the value of labour power. Matsuo [7] defines the labour value of a vector $b$ referring to the notion of Minimised Labour for Equal Utility (MLEU): the labour value of bundle $b$ corresponds to the minimum amount of labour necessary to produce another bundle $c$ as net output, which gives at least the same utility as $b$. Formally, let $\mathcal{C}$ denote the set of continuous functions. For the sake of simplicity, and without loss of generality, assume that leisure does not enter the workers' utility functions, so that for every agent $\nu \in W, u^{\nu}: \mathbb{R}_{+}^{n} \rightarrow \mathbb{R}$ is the utility function representing worker $\nu$ 's preference over consumption. The equivalent of Matsuo's ([7]) Assumption 2 in a general framework with heterogeneous agents can then be written as follows. ${ }^{4}$

Assumption (A4). For each $\nu \in W, u^{\nu} \in \mathcal{U}$, where $\mathcal{U}=\left\{u^{\nu} \in \mathcal{C} \mid c^{\prime} \geq c \Rightarrow\right.$ $\left.u^{\nu}\left(c^{\prime}\right)>u^{\nu}(c)\right\}$.

In other words, each worker's utility function is continuous and strictly increasing (in the first $n$ arguments).

Let us introduce Matsuo's notion of Minimised Labour for Equal Utility (MLEU) as follows.

Definition 1: For a given $u^{\nu} \in \mathcal{U}$, the labour value of vector $b$ according to

\footnotetext{
${ }^{4}$ Leisure is not included in utility functions for notational simplicity and conceptual clarity. First, although [7] assumes that workers have preferences over leisure, this assumption plays no role at all in his argument and indeed he imposes no restriction whatsoever concerning the effect of leisure on welfare. Second, if leisure is included in the utility function some conceptual issues arise concerning the definition of labour value (see the next footnote). Finally, the introduction of leisure in the utility functions would leave all the theoretical arguments and formal results in this paper unchanged.
} 
the MLEU view, relative to agent $\nu$, is the solution of the following problem:

$$
M L^{\nu}: \min _{\alpha=\left(-\alpha_{0},-\underline{\alpha}, \bar{\alpha}\right) \in P} \alpha_{0} \text { s.t. } \bar{\alpha}-\underline{\alpha} \geqq c\left(\forall c \in \mathbb{R}_{+}^{n}: u^{\nu}(c) \geqq u^{\nu}(b)\right) .
$$

Denote the solution of the above problem by $\alpha^{u^{\nu}}(b)$ : the labour value of $b$ w.r.t. $u^{\nu}$ is defined as $\alpha_{0}^{u^{\nu}}(b) .^{5}$ Two crucial properties of Matsuo's subjectivist definition of the labour content of a bundle $b$ are immediately apparent from Definition 1: first, the concept of labour value depends on subjective preferences, and if agents are heterogenous in principle there is no unique value of $b .^{6}$ Second, in this approach, the notion of labour value becomes more and more abstract and far from the productive conditions of the economy.

Based on Definition 1, the concept of MLEU-exploitation of an agent $\nu$ consuming $b^{\nu}$ and supplying $l$ can be specified.

Definition 2: (i) Given $u^{\nu} \in \mathcal{U}$, worker $\nu \in W$ is MLEU-exploited w.r.t. $u^{\nu}$ if and only if $l-\alpha_{0}^{u^{\nu}}\left(b^{\nu}\right)>0$. (ii) Further, worker $\nu \in W$ is MLEUexploited if and only if $l-\alpha_{0}^{u^{\nu}}\left(b^{\nu}\right)>0$ for all $u^{\nu} \in \mathcal{U}$.

If a representative agent is assumed, $u^{\nu}=u$ for all $\nu \in W$, and Definitions 1 and 2 should be modified accordingly by replacing $\alpha_{0}^{u^{\nu}}(b)$ with $\alpha_{0}^{u}(b)$.

Definitions 1 and 2 represent the core of Matsuo's approach. Because the condition in Definition 2(ii) must hold for all $u^{\nu} \in \mathcal{U}$, and the existence of exploitation can be proved regardless of the specific $u^{\nu}$, he maintains that "This causes this condition to be objective" ([7], p.260). This claim is misleading: although the existence of exploitation may be independent of

\footnotetext{
${ }^{5}$ In [7] leisure is included in workers' utility functions, which are also assumed to be identical, and thus the relevant constraint in $M L$ is written as $u(c, l) \geq u(b, l)$, where $l$ is the amount of labour expended by workers to be able to buy $b$. As already noted, the inclusion of labour has no relevance for the formal results. Yet, from a theoretical viewpoint, it seems arbitrary to keep labour constant at $l$ in the left-hand-side of the constraint. It is not at all clear why the amount of labour in workers' utility functions should remain constant even at the new allocation $c$.

${ }^{6}$ Interestingly, in the economy with $P=P_{(A, B, L)}$, Matsuo ([7], Definition 3) defines a "Narrow Effective Range of Value" as the set of strictly positive vectors $t$ such that $t(B-A) \leqq L$. The vectors $t$ seem the generalisation of the standard vector of embodied labour $t=L(I-A)^{-1}$ of the Leontief technology, and they only depend on the objective features of the economy relating to the conditions of production. In this framework, it would then seem natural to define the labour value of a bundle $c$ as $t c$. Yet they play no essential role in Matsuo's ([7]) analysis.
} 
the specific $u^{\nu} \in \mathcal{U}$, the labour value of a bundle does depend on the specific $u^{\nu}$ and the value of labour power cannot be defined without knowing $u^{\nu}$.

If workers are heterogeneous, the inherently subjective dimension of Matsuo's approach, and a number of problematic features, are particularly clear when it comes to the construction of an aggregate index of exploitation. Consider an economy with preferences $\left(u^{\nu}\right)_{\nu \in W}$ and consumption bundles $\left(b^{\nu}\right)_{\nu \in W}$. Let $\beta \equiv \sum_{\nu \in W} b^{\nu}$ denote aggregate workers' consumption. Suppose, for the sake of argument, that an aggregate subjectivist exploitation index could be constructed: if agents are heterogeneous and consume different bundles, a permutation of their bundles, leaving $\beta$ and the preference profile unchanged, will lead in general to changes in the individual and aggregate exploitation indices. This is extremely counterintuitive in exploitation theory, especially given that workers work the same amount of time and earn the same income.

More importantly, it is unclear that a meaningful index can be constructed in the general case: the aggregate level of exploitation cannot be consistently determined by knowing only $\beta$, because there is an inherent indeterminacy in the definition of the economy-wide labour value of $\beta$. Therefore one would have to start from individual exploitation indices (or the individual $\alpha_{0}^{u^{\nu}}\left(b^{\nu}\right)$ 's) and find a consistent way of aggregating them. Yet, while the individual index of exploitation of worker $\nu$, who works $l$ and consumes $b^{\nu}$, relative to $u^{\nu}$, can be defined as $e^{u^{\nu}}\left(b^{\nu}, l\right)=\frac{l-\alpha_{0}^{u^{\nu}}\left(b^{\nu}\right)}{l}$, there is no obvious way of aggregating the different indices $e^{u^{\nu}}\left(b^{\nu}, l\right)$ into an economy-wide measure of exploitation, which is denoted by $e\left(\left(b^{\nu}\right)_{\nu \in W}, l,\left(u^{\nu}\right)_{\nu \in W}\right)$. Actually, this is true even if all workers consume the same bundle, and some deep ambiguity seems inherent in this subjectivist approach, unless a representative worker is assumed, in which case $e(b, l, u)=e^{u}(b, l)$.

These problems undermine the positive and normative significance of Matsuo's subjectivist approach. To be sure, one may object that the main purpose of exploitation theory is to diagnose the existence of exploitation, whereas the construction of an exploitation index is not essential. This defence is unconvincing, because it implies that it is impossible to compare different societies based on the amount of exploitation suffered by workers, nor is it meaningful to analyse the dynamics of exploitation of a society over time.

Indeed, even if a representative agent is assumed, so that a subjectivist exploitation index can be defined, not much can be said about the degree of 
exploitation in an economy, apart from diagnosing its existence. Theorem 1 precisely characterises this deep, additional indeterminacy. As a preliminary step, let $\mathcal{E}_{H} \subset \mathcal{E}$ be the subset of economies such that any $E \in \mathcal{E}_{H}$ has a representative agent. Moreover, $\mathcal{E}_{H}\left(\left\langle K, W ; P ;\left(\omega^{\nu}\right)_{\nu \in K}\right\rangle\right) \subset \mathcal{E}_{H}$ denotes a subset of economies with the same objective features, $\left\langle K, W ; P ;\left(\omega^{\nu}\right)_{\nu \in K}\right\rangle$, and a representative agent but potentially different preferences.

Theorem 1: (i) For any $E \in \mathcal{E}_{H}, \alpha_{0}^{u}(b)=\bar{l}$ for all $u \in \mathcal{U}$ if and only if $\{b\}=S \widehat{P}\left(\alpha_{0}=\bar{l}\right)$.

(ii) There is a set $\mathcal{E}_{H}\left(\left\langle K, W ; P ;\left(\omega^{\nu}\right)_{\nu \in K}\right\rangle\right) \subset \mathcal{E}_{H}$ such that for all $\delta>0$ and all $\varepsilon \in[0,1]$ there is a function $u \in \mathcal{U}$, such that $\left|e^{u}(b, l)-\varepsilon\right|<\delta$.

Proof. Part (i). First, if $\{b\}=S \widehat{P}\left(\alpha_{0}=\bar{l}\right)$, then for all $\alpha^{\prime} \in P$ such that $\alpha_{0}^{\prime} \leqq \alpha_{0}=\bar{l}, \widehat{\alpha}^{\prime} \leqq \widehat{\alpha}=b$ and if $\alpha_{0}^{\prime}<\alpha_{0}$, then $\widehat{\alpha}^{\prime} \leq \widehat{\alpha}$. But then, (A4) implies that $\alpha_{0}^{u}(b)=\bar{l}$ for all $u \in \mathcal{U}$. Conversely, if $b \notin S \widehat{P}\left(\alpha_{0}=\bar{l}\right)$, then obviously $\alpha_{0}^{u}(b) \neq \bar{l}$ for some $u \in \mathcal{U}$. Suppose that there is $b^{\prime} \neq b$ such that $\left\{b, b^{\prime}\right\} \subset S \widehat{P}\left(\alpha_{0}=\bar{l}\right)$. Then, there exist $i, j: b_{i}^{\prime}>b_{i}$ and $b_{j}^{\prime}<b_{j}$. Further, $\widetilde{b}=\lambda b+(1-\lambda) b^{\prime} \in S \widehat{P}\left(\alpha_{0}=\bar{l}\right)$ for all $\lambda \in[0,1]$, since $P$ is convex. Consider $\mathcal{U}^{p} \equiv\left\{u \in \mathcal{U} \mid u(c)=\sum_{i=1}^{n} \delta_{i} c_{i}, \delta_{i}>0, \sum_{i=1}^{n} \delta_{i}=1\right\}$. There is always $u \in$ $\mathcal{U}^{p}$ such that $u(\widetilde{b})>u(b)$ for any $\lambda \in(0,1]$, and therefore $\alpha_{0}^{u}(b)<\bar{l}$.

Part (ii). Let $\mathcal{E}_{H}\left(\left\langle K, W ; P ;\left(\omega^{\nu}\right)_{\nu \in K}\right\rangle\right)$ be defined by $K=\{\nu\}, W=\{\mu\}$, $\omega^{\nu}=\left[\begin{array}{l}1 \\ 1\end{array}\right]$, and $P=P_{(A, B, L)}$ with

$$
B=\left[\begin{array}{ll}
1 & 3 \\
2 & 2
\end{array}\right], A=\left[\begin{array}{ll}
1 & 1 \\
1 & 1
\end{array}\right], \text { and } L=(1,1)
$$

Let $b=\left[\begin{array}{l}2 \\ 0\end{array}\right]$ and $l=1$. Then, as in part (i), consider $\mathcal{U}^{p}$ and define the infinite sequence $\left\{u^{t}(c)\right\}_{t=0}^{\infty} \subset \mathcal{U}^{p}$ with $u^{t}(c) \equiv \delta^{t} c_{1}+\left(1-\delta^{t}\right) c_{2}$, where $\delta^{t} \in(0,1)$, for all $t$. Since $e^{u^{t}}(b, 1)=1-\alpha_{0}^{u^{t}}(b), \alpha_{0}^{u^{t}}(b) \rightarrow 0$ as $\delta^{t} \rightarrow 0$, and thus $e^{u^{t}}(b, 1) \rightarrow 1$, whereas $\alpha_{0}^{u^{t}}(b) \rightarrow 1$ as $\delta^{t} \rightarrow 1$, and thus $e^{u^{t}}(b, 1) \rightarrow 0$.

Remark 1 It is worth noting that $p=(0.5,0.5)$ and $x=\left[\begin{array}{l}0 \\ 1\end{array}\right]$ constitute a Reproducible Solution as defined by Roemer ([13] and [14]; see also the next section) associated with $\pi=\frac{1}{2}$. At this reproducible solution, $A x=\omega$, $(B-A) x \geq b, L x=1$, and $p b=1$. 
By Theorem 1(i), even if workers are identical, and thus no aggregation issue arises, the exploitation index will be invariant to changes in workers' preferences only in the rather special case that there exists a certain amount of labour input such that the wage basket lies on the corresponding production possibility frontier, and the latter corresponds to a single point. Theorem 1(ii) has an even more puzzling implication: even if workers are identical, there are economies in which it is literally impossible in principle to say anything about exploitation, except whether it exists. In fact, for a given set of objective characteristics of the economy, the amount of exploitation suffered by each worker can take any value provided the appropriate utility function is chosen. By simply changing workers' subjective preferences, the economy moves from being essentially non-exploitative, to being plagued by the most extreme form of exploitation. In this kind of situation, the exploitation index is not just inaccurate, it is meaningless. ${ }^{7}$

By focusing on a simple von Neumann technology, the following example forcefully illustrates the implications of Theorem 1, if the condition in Part (i) is violated, so that there exist two economies $E, E^{\prime} \in \mathcal{E}_{H}$, such that $K=K^{\prime}, L=L^{\prime},\left(P_{(A, B, L)}, b\right)=\left(P_{(A, B, L)}^{\prime}, b^{\prime}\right),\left(\omega^{\nu}\right)_{\nu \in K}=\left(\omega^{\prime \nu}\right)_{\nu \in K}$, but $e^{u}(b, l) \neq e^{u^{\prime}}(b, l)$.

Example 1: Consider the following von Neumann production technology $(A, B, L)$ and bundle of wage goods $b$ :

$$
B=\left[\begin{array}{ll}
2 & 3 \\
2 & 2
\end{array}\right], A=\left[\begin{array}{ll}
1 & 1 \\
1 & 1
\end{array}\right], L=(1,1), \text { and } b=\left[\begin{array}{l}
1 \\
1
\end{array}\right] .
$$

Let $K=\{\nu\}, W=\{\mu\}$, and $\omega^{\nu}=\left[\begin{array}{l}1 \\ 1\end{array}\right]$. It can easily be shown that $p=(0.5,0.5)$ and $x=\left[\begin{array}{l}0 \\ 1\end{array}\right]$ constitute a Reproducible Solution as defined by Roemer ([13] and [14]; see also the next section) associated with the maximal profit rate $\pi=\frac{1}{2}$. Moreover, $[B-A] x \geqq b L x$ and $A x \leqq \omega$. Finally, $p b=1$.

\footnotetext{
${ }^{7}$ It is worth noting in passing that Matsuo defends his subjectivist approach by arguing that "exploitation is a matter of alienation" ([7], p.263), that is, it derives from the workers' "exclusion from decision making on the production allocation of the society" ([7], p.263). This argument seems false, for in Matsuo's framework, exploitation would be eliminated if capitalists continued to organise production but workers received the whole of net product. It is also arguably misleading, if not conceptually inappropriate, to conflate two distinct phenomena.
} 
Let $u \in \mathcal{U}$ be such that, for any $y^{\prime}=\left(y_{1}^{\prime}, y_{2}^{\prime}\right) \in \mathbb{R}_{+}^{2}, u\left(y^{\prime}\right)=y_{1}^{\prime} \cdot y_{2}^{\prime}$. It follows that $b=\arg \max _{p y^{\prime}=1} u\left(y^{\prime}\right)$. Thus, if $u$ is interpreted as representing standard subjective preferences over consumption (as in the neoclassical theory of consumer behavior), the vector $b$ can be interpreted as the worker's Marshallian demand which is purchased under the budget constraint $p y^{\prime}=1$. In contrast, it follows from Definition 1 that $\alpha_{0}^{u}(b)=L \widetilde{x}=\frac{\sqrt{2}}{2}$ with $\widetilde{x}=\left(\widetilde{x}_{1}, \widetilde{x}_{2}\right)=\left(0, \frac{\sqrt{2}}{2}\right)$, and $u(b)=u(\widetilde{y})$ holds for $\widetilde{y} \equiv[B-A] \widetilde{x}=$ $\left(\sqrt{2}, \frac{\sqrt{2}}{2}\right)$. Hence, since $1-L \widetilde{x}>0$, the worker is MLEU-exploited w.r.t. $u$. Next, let $u^{\prime} \in \mathcal{U}$ be given as:

$$
\frac{\partial u^{\prime}(b)}{\partial b_{1}} / \frac{\partial u^{\prime}(b)}{\partial b_{2}}=1 \& \forall y^{\prime}(\neq b) \in \mathbb{R}_{+}^{2} \text { with } u\left(y^{\prime}\right)=u(b), u^{\prime}\left(y^{\prime}\right)<u^{\prime}(b) \text {. }
$$

Insert Figures 1 and 2 around here.

In other words, $U(b ; u) \supsetneq U\left(b ; u^{\prime}\right)$. In this case, $b=\arg \max _{p y^{\prime}=1} u^{\prime}\left(y^{\prime}\right)$ holds. Thus, again, if we interpret $u^{\prime}$ as representing a standard subjective preference of the worker over consumption such as in the neoclassical theory of consumers behavior, the vector $b$ can be interpreted as the worker's Marshallian demand which is purchased under the budget constraint $p y^{\prime}=1$. Note that it can easily be shown that $1-L \widetilde{x}^{\prime}>0$ still holds, and so the worker is MLEU-exploited w.r.t. $u^{\prime}$. However, now, $\alpha_{0}^{u^{\prime}}(b)=L \widetilde{x}^{\prime}>\frac{\sqrt{2}}{2}$ holds, since by construction $u^{\prime}(\widetilde{y})<u^{\prime}(b)$ for $\widetilde{y}=\left(\sqrt{2}, \frac{\sqrt{2}}{2}\right)$, so that $\widetilde{y}^{\prime} \equiv[B-A] \widetilde{x}^{\prime}>\widetilde{y}$. Hence, although the worker's conditions are unchanged, because she provides one unit of labour and receive one unit of wage revenue per day, her 'exploitation rate' would decrease if her subjective preferences changed from $u$ to $u^{\prime}$.

\section{The subjectivist approach and the FMT}

In this section, the assumption $l=1$ is made, without loss of generality. Matsuo [7] insists that exploitation derives from the workers' lack of control over production processes, and if workers could access all production processes, they would not be exploited and would reach a higher utility. According to this scenario, he defines the following maximisation problem:

$$
\max _{\alpha=\left(-\alpha_{0},-\underline{\alpha}, \bar{\alpha}\right) \in P} u^{\nu}(\widehat{\alpha}) \text { s.t. } \widehat{\alpha} \in \mathbb{R}_{+}^{n} \text { and } \alpha_{0} \leqq 1 .
$$


The solution of the above problem can be denoted by $\alpha_{\max }^{u^{\nu}}$, and its corresponding utility value by $\bar{u}_{\max }^{u^{\nu}}$, which reduce to $\alpha_{\max }^{u}$ and $\bar{u}_{\max }^{u}$, respectively, if a representative agent is assumed.

Theorem 2 generalises Matsuo's ([7]) 'Weak System of Exploitation Theory' (WSET) in two important directions: first, it allows for heterogenous workers' preferences, even if workers consume a given subsistence bundle; second, the equivalence results are shown to hold in general convex cone economies.

Theorem 2 (The Generalised WSET): For any economy $E \in \mathcal{E}$, where $b$ is the wage bundle, the following statements are equivalent:

(1) $b \in \widehat{P}\left(\alpha_{0}=1\right) \backslash S \widehat{P}\left(\alpha_{0}=1\right)$;

(2) For each $\nu \in W, 1-\alpha_{0}^{u^{\nu}}(b)>0$ holds for each $u^{\nu} \in \mathcal{U}$;

(3) For each $\nu \in W, u^{\nu}(b)<\bar{u}_{\max }^{u^{\nu}}$ holds for each $u^{\nu} \in \mathcal{U}$;

(4) $\nexists p \in \mathbb{R}_{++}^{n}$ s.t. $p[\widehat{\alpha}-b] \leqq 0$ holds for all $\widehat{\alpha} \in S \widehat{P}\left(\alpha_{0}=1\right)$.

Proof. 1. First, we prove that $(1) \Leftrightarrow(2)$.

$(\Rightarrow)$ : Let $b \in \widehat{P}\left(\alpha_{0}=1\right) \backslash S \widehat{P}\left(\alpha_{0}=1\right)$. Then, by definition of $\widehat{P}\left(\alpha_{0}=1\right)$, it needs at most one unit of labour to produce $b$ as a net output. Since $b \in \widehat{P}\left(\alpha_{0}=1\right) \backslash S \widehat{P}\left(\alpha_{0}=1\right)$, there exists $\alpha \in P$ such that $\widehat{\alpha} \in S \widehat{P}\left(\alpha_{0}=1\right)$ and $\widehat{\alpha} \geq b$. Then, for each $u^{\nu} \in \mathcal{U}, u^{\nu}(\widehat{\alpha})>u^{\nu}(b)$ holds. Then, we can find $c \in \widehat{P}\left(\alpha_{0}=1\right) \backslash S \widehat{P}\left(\alpha_{0}=1\right)$ such that $\widehat{\alpha} \geq c \geq b$. Then, again, for any $u^{\nu} \in \mathcal{U}, u^{\nu}(c)>u^{\nu}(b)$ holds, and $c \in U\left(b ; u^{\nu}\right)$. Since $U\left(b ; u^{\nu}\right)$ is an open set for each $u^{\nu}$, then there is an open neighbourhood $N(c)$ of $c$ such that $N(c) \subseteq U\left(b ; u^{\nu}\right)$. Thus, there is $c^{\prime} \in \stackrel{\circ}{P}\left(\alpha_{0}=1\right)$ such that $c^{\prime} \in U\left(b ; u^{\nu}\right)$ and there is $\alpha^{\prime} \in P$ such that $\bar{\alpha}^{\prime}-\underline{\alpha}^{\prime} \geq c^{\prime}$ and $\alpha_{0}^{\prime}<1$. Hence it follows from Definition 1 that $1-\alpha_{0}^{u^{\nu}}(b)>0$. This reasoning holds for each $u^{\nu} \in \mathcal{U}$.

$(\Leftarrow)$ : Suppose $b \in S \widehat{P}\left(\alpha_{0}=1\right)$. Then, there exists a suitable $u^{\nu} \in \mathcal{U}$ which satisfies $U\left(b ; u^{\nu}\right) \cap \widehat{P}\left(\alpha_{0}=1\right)=\varnothing$. This implies, by the continuity of $u^{\nu}, \alpha_{0}^{u^{\nu}}(b)=1$. Suppose $b \notin \widehat{P}\left(\alpha_{0}=1\right)$. Then, again, there exists a suitable $u^{\nu} \in \mathcal{U}$ which satisfies $U\left(b ; u^{\nu}\right) \cap \widehat{P}\left(\alpha_{0}=1\right)=\varnothing$, which implies $\alpha_{0}^{u^{\nu}}(b) \geqq 1$.

2. Next, we prove that $(1) \Leftrightarrow(3)$.

$(\Rightarrow)$ : Let $b \in \widehat{P}\left(\alpha_{0}=1\right) \backslash S \widehat{P}\left(\alpha_{0}=1\right)$. Then, for any $u^{\nu} \in \mathcal{U}$, there exists $c^{u^{\nu}} \in S \widehat{P}\left(\alpha_{0}=1\right)$ such that $u^{\nu}\left(c^{u^{\nu}}\right)>u^{\nu}(b)$ holds. Note, for any $u^{\nu} \in \mathcal{U}$, $\bar{u}_{\max }^{u^{\nu}} \geqq u^{\nu}\left(c^{u^{\nu}}\right)$ holds. Thus, for any $u^{\nu} \in \mathcal{U}, u^{\nu}(b)<\bar{u}_{\max }^{u^{\nu}}$.

$(\Leftarrow)$ : Suppose $b \in S \widehat{P}\left(\alpha_{0}=1\right)$. Then, there exists a suitable $u^{\nu} \in \mathcal{U}$ such that $\bar{u}_{\max }^{u^{\nu}}=u^{\nu}(b)$. Suppose $b \notin \widehat{P}\left(\alpha_{0}=1\right)$. Then, there exists a 
suitable $u^{\nu} \in \mathcal{U}$ which satisfies $U\left(b ; u^{\nu}\right) \cap \widehat{P}\left(\alpha_{0}=1\right)=\varnothing$, which implies $u^{\nu}(b) \geqq \bar{u}_{\max }^{u^{\nu}}$.

3. Finally, we prove that $(1) \Leftrightarrow(4)$.

$(\Rightarrow)$ : Let $b \in \widehat{P}\left(\alpha_{0}=1\right) \backslash S \widehat{P}\left(\alpha_{0}=1\right)$. Then there is $\alpha \in P$ such that $\widehat{\alpha} \in S \widehat{P}\left(\alpha_{0}=1\right)$ and $\widehat{\alpha} \geq b$ and $p[\widehat{\alpha}-b]>0$ for all $p \in \mathbb{R}_{++}^{n}$.

$(\Leftarrow)$ : If $b \in S \widehat{P}\left(\alpha_{0}=1\right)$, there is $p \in \mathbb{R}_{++}^{n}$ such that for all $\widehat{\alpha} \in$ $S \widehat{P}\left(\alpha_{0}=1\right), p[\widehat{\alpha}-b] \leqq 0$, a contradiction. If $b \notin \widehat{P}\left(\alpha_{0}=1\right)$, let $X(b) \equiv$ $\left\{\widehat{\alpha}-b \mid \widehat{\alpha} \in \widehat{P}\left(\alpha_{0}=1\right)\right\}$. Since $b \notin \widehat{P}\left(\alpha_{0}=1\right)$, then $X(b) \cap \mathbb{R}_{+}^{n}=\varnothing$. In fact, if there exists $x \in X(b) \cap \mathbb{R}_{+}^{n}$, this implies that there is $\widehat{\alpha}^{\prime} \in \widehat{P}\left(\alpha_{0}=1\right)$ such that $\widehat{\alpha}^{\prime}-b=x \geqq 0$. Then, by A3, $b \in \widehat{P}\left(\alpha_{0}=1\right)$ holds, which is a contradiction. Thus, by the separating hyperplane theorem, there is $p^{*} \in \mathbb{R}^{n} \backslash\{\mathbf{0}\}$ such that for any $x=\widehat{\alpha}-b \in X(b), p^{*}[\widehat{\alpha}-b]<0$. Moreover, since the hyperplane associated with $p^{*}$ separates $X(b)$ and $\mathbb{R}_{+}^{n}, p^{*} \geq \mathbf{0}$ holds by Nikaido [11; Theorem 30.1]. If $p^{*} \notin \mathbb{R}_{++}^{n}$, take another $p^{\prime} \in \mathbb{R}_{++}^{n}$ which is sufficiently close to $p^{*}$. Then, $p^{\prime}[\widehat{\alpha}-b]<0$ still holds for all $\widehat{\alpha} \in \widehat{P}\left(\alpha_{0}=1\right)$, since $p[\widehat{\alpha}-b]$ is continuous at $p^{*}$ for each $\widehat{\alpha} \in \widehat{P}\left(\alpha_{0}=1\right)$, and a contradiction obtains.

Theorem 2 proves that (2) every worker in the economy is MLEUexploited if and only if (1) her consumption bundle $b$ can be produced with less than the one unit of labour that she has supplied. In turn, the latter holds if and only if (3) workers do not get their maximum utility (for any continuous and strictly increasing utility function). All these conditions are equivalent to (4) the existence of strictly positive profits for each strictly positive price vector. Matsuo's main Theorem follows as a corollary of Theorem 2 , in the special case of von Neumann technology and identical preferences.

Corollary 1: For any economy $E \in \mathcal{E}$, with $P=P_{(A, B, L)}$ for some $(A, B, L)$, the following statements are equivalent:

(1) $\nexists p \in \mathbb{R}_{++}^{n}$ s.t. $p[B-A-b L] \leqq 0$;

(2) $\exists x \in \mathbb{R}_{+}^{m}$ s.t. $[B-A-b L] x \geq 0$;

(3) For each $\nu \in W, 1-\alpha_{0}^{u^{\nu}}(b)>0$ holds for each $u^{\nu} \in \mathcal{U}$;

(4) For each $\nu \in W, u^{\nu}(b)<\bar{u}_{\max }^{u^{\nu}}$ holds for each $u^{\nu} \in \mathcal{U}$.

Theorem 2 does generalise the core result of Matsuo's subjectivist approach, but it also highlights its limits and problematic implications. First, 
Theorem 2 does not hold if workers are allowed to consume different bundles. In particular, for a given $\beta=\sum_{\nu \in W} b^{\nu}$, if the assumption $b^{\nu}=b$ for all $\nu \in W$ is dropped, then even if $\frac{\beta}{N} \in \widehat{P}\left(\alpha_{0}=1\right) \backslash S \widehat{P}\left(\alpha_{0}=1\right)$, conditions (2) and (3) do not necessarily hold. The equivalence is restored if condition (1) is written as $b^{\nu} \in \widehat{P}\left(\alpha_{0}=1\right) \backslash S \widehat{P}\left(\alpha_{0}=1\right)$ for all $\nu \in W$. But the latter condition is theoretically ad hoc and empirically questionable as it focuses on the production conditions of arbitrary individual wage bundles.

In principle, one might try to restore the equivalence in Theorem 2 by replacing the analysis of individual workers in (2) and (3) with a focus on some aggregate exploitation index. As argued above, however, in general economies with heterogeneous workers consuming different bundles, it is difficult to construct an aggregate exploitation index that is meaningful and fully consistent with strong subjectivism. Therefore it is unclear that a strongly subjectivist approach can actually deal with general economies with heterogeneous agents.

Alternatively, one might insist that, even if workers have different preferences, it is consistent with the traditional Marxian view to assume that they consume the same subsistence bundle. Or perhaps, one might argue that a representative agent framework is theoretically appropriate in exploitation theory. In either case, Theorem 2 does generalise the WSET, even though it is important to note that the representative agent assumption is not an innocuous technical condition. Yet, even in these simplified cases, it is unclear that this subjectivist approach provides a satisfactory treatment of the FMT. Theorem 2 only focuses on strictly positive price vectors, but this is rather restrictive, as there are many cases in which the equilibrium price vector is only semipositive. Proposition 1 proves that, for any semipositive price vector if profits are positive, then the economy is MLEU-exploitative.

Proposition 1: For any economy $E \in \mathcal{E}$, if $((p, 1), \alpha)$ is a pair of a semipositive price vector and a social production point such that $\widehat{\alpha} \geqq \alpha_{0} b$, for a given wage bundle $b$, and profits are positive, then $1-\alpha_{0}^{u^{\nu}}(b)>0$, for all $\nu \in W$, for any $\left(u^{\nu}\right)_{\nu \in W}$ such that $u^{\nu} \in \mathcal{U}$.

Proof. Let $((p, 1), \alpha)$ be a price vector and a social production point such that $\widehat{\alpha} \geqq \alpha_{0} b$ and $p \widehat{\alpha}-\alpha_{0}>0$. Let $\alpha^{*} \equiv \alpha / \alpha_{0}$. Then, $p \widehat{\alpha}^{*}-1>0$ and $\widehat{\alpha}^{*} \geqq b$. By definition, $\widehat{\alpha}^{*} \in \widehat{P}\left(\alpha_{0}=1\right)$. Since $\widehat{\alpha}^{*} \geqq b$, it follows that $b \in \widehat{P}\left(\alpha_{0}=1\right) \backslash S \widehat{P}\left(\alpha_{0}=1\right)$ or $\widehat{\alpha}^{*}=b$. Since $p \widehat{\alpha}^{*}-1>0$ for $p b=1, \widehat{\alpha}^{*}=b$ is impossible, so that $b \in \widehat{P}\left(\alpha_{0}=1\right) \backslash S \widehat{P}\left(\alpha_{0}=1\right)$ holds. By Theorem 2 , the 
desired result is obtained.

Theorem 2 , however, does not rule out the possibility that $p[\widehat{\alpha}-b] \leqq 0$ holds for some $p \geq 0$ even when condition (2) holds, i.e. that exploitation occurs without positive profits, contradicting the FMT. The next two results establish that the FMT is indeed violated under two standard definitions of equilibrium.

Consider, first, von Neumann's concept of balanced growth equilibrium. Assume wages to be advanced and let $p b=1$.

Definition 3 [17]: $A$ balanced growth equilibrium (BGE) for the economy $E \in \mathcal{E}$ with $P=P_{(A, B, L)}$ is a tuple $((p, 1), x, \pi)$, where $p \in \mathbb{R}_{+}^{n}, x \in \mathbb{R}_{+}^{m}$, and $\pi>-1$ such that:

(a) $p B \leqq(1+\pi)[p A+L]$;

(b) $B x \geqq(1+\pi)[A+b L] x$;

(c) $p B x>0$.

In Definition 3, (a) is the revenue-cost condition for each production process in equilibrium, which implies that, given competition among production processes, in equilibrium no capitalist can gain more than the warranted profit rate $\pi$ from operating any production process. Note that the warranted profit rate $\pi$ is the minimal value of the (uniform) profit rate warranted for all production processes in equilibrium. In contrast, (b) is the demand and supply condition for each capital and/or consumption good, which implies that in the equilibrium, the demand of any capital or consumption goods used for the next production period, $(1+\pi)[A+b L] x$, does not exceed the supply of those goods, $B x$, produced in this period. Here, $\pi$ represents the maximum growth rate of the economy. Finally, condition (c) implies that the total market value of output should be positive, which eliminates trivial equilibria with no production.

The next result proves that the FMT does not hold in Matsuo's subjectivist approach, in the von Neumann balanced growth equilibrium.

Theorem 3: There is an economy $E \in \mathcal{E}$ with $P=P_{(A, B, L)}$ and $b^{\nu}=b$ for all $\nu$, in which at any $\mathrm{BGE}$, the corresponding warranted profit rate is zero while MLEU-exploitation exists.

Proof. 1. Consider a von Neumann production technology $(A, B, L)$ and a bundle of wage goods $b$ as follows: 


$$
B=\left[\begin{array}{ll}
2 & 3 \\
2 & 2
\end{array}\right], A=\left[\begin{array}{ll}
1 & 1 \\
1 & 1
\end{array}\right], L=(1,1), \text { and } b=\left[\begin{array}{l}
1 \\
1
\end{array}\right]
$$

In this case, regardless of the distribution of capital endowment $\left(\omega^{\nu}\right)_{\nu \in K}$, the set of BGEs - normalised so that $L x=1$ - is given by

$$
B G E_{(A, B, L, b)} \equiv\{(0,1), 1\} \times\left\{\left(x_{1}^{\prime}, x_{2}^{\prime}\right) \in \mathbb{R}_{+}^{2} \mid x_{1}^{\prime}+x_{2}^{\prime}=1\right\} \times\{0\}
$$

To show this, let $p=(0,1)$. First of all, note that $p b=1$. Then, since $p[B-A]=(1,1)$ and $\pi p A+(1+\pi) L=\pi(1,1)+(1+\pi)(1,1)$, the warranted profit rate at this price is $\pi=0$. Moreover, since $[B-A] x=$ $\left[\begin{array}{l}x_{1}+2 x_{2} \\ x_{1}+x_{2}\end{array}\right]$ and $\pi A x+(1+\pi) b L x=\pi\left[\begin{array}{l}x_{1}+x_{2} \\ x_{1}+x_{2}\end{array}\right]+(1+\pi)\left[\begin{array}{l}x_{1}+x_{2} \\ x_{1}+x_{2}\end{array}\right]$, it follows that since $\pi=0$ then Definition 3(b) holds. Finally, for any $((p, 1), x, \pi) \in B G E_{(A, B, L, b)}, p B x>0$, so that Definition 3(c) holds. Thus, if $((p, 1), x, \pi) \in B G E_{(A, B, L, b)}$, then it constitutes a BGE.

2. Let us examine whether there is another BGE. First of all, it is immediate to show that if $\left(\left(p^{\prime \prime}, 1\right), x^{\prime \prime}, \pi^{\prime \prime}\right)$ constitutes a BGE, then $\pi^{\prime \prime}=0$ must hold from Definition 3(b). Then, $p^{\prime \prime}[B-A]=\left(p_{1}^{\prime \prime}+p_{2}^{\prime \prime}, 2 p_{1}^{\prime \prime}+p_{2}^{\prime \prime}\right)$ and $\pi^{\prime \prime} p^{\prime \prime} A+\left(1+\pi^{\prime \prime}\right) L=(1,1)$. Since $p_{1}^{\prime \prime}+p_{2}^{\prime \prime}=1$ by $p b=1$, it follows that $p^{\prime \prime}[B-A]=\left(1, p_{1}^{\prime \prime}+1\right)$. Thus, if $\left(\left(p^{\prime \prime}, 1\right), x^{\prime \prime}, \pi^{\prime \prime}\right)$ constitutes a BGE, $p_{1}^{\prime \prime}=0$ holds from Definition 3(a). Therefore, if $\left(\left(p^{\prime \prime}, 1\right), x^{\prime \prime}, \pi^{\prime \prime}\right)$ constitutes a BGE, then $\left(\left(p^{\prime \prime}, 1\right), x^{\prime \prime}, \pi^{\prime \prime}\right) \in B G E_{(A, B, L, b)}$.

3. The above argument implies that in this economy, the warranted profit rate is zero at every BGE. Hence, if Definition 2 is adopted and the FMT holds in this economy, then there should be no MLEU-exploitation. However, recalling that

$P_{(A, B, L)} \equiv\left\{\left(-\alpha_{0},-\underline{\alpha}, \bar{\alpha}\right) \in \mathbb{R}_{-}^{n+1} \times \mathbb{R}_{+}^{n} \mid \exists x \in \mathbb{R}_{+}^{m}: \alpha_{0}=L x \&(-\underline{\alpha}, \bar{\alpha}) \leqq(-A x, B x)\right\}$, it follows that $\widehat{P}\left(\alpha_{0}=1\right)=c o\left\{\left[\begin{array}{l}2 \\ 0\end{array}\right],\left[\begin{array}{l}2 \\ 1\end{array}\right],\left[\begin{array}{l}0 \\ 1\end{array}\right],\left[\begin{array}{l}0 \\ 0\end{array}\right]\right\}$ and $S \widehat{P}\left(\alpha_{0}=1\right)=\left\{\left[\begin{array}{l}2 \\ 1\end{array}\right]\right\}$, and therefore $b=\left[\begin{array}{l}1 \\ 1\end{array}\right] \in \widehat{P}\left(\alpha_{0}=1\right) \backslash S \widehat{P}\left(\alpha_{0}=1\right)$. Hence, by Theorem 2, for any $u^{\nu} \in \mathcal{U}, 1-\alpha_{0}^{u^{\nu}}(b)>0$. This implies that MLEU-exploitation exists and the FMT does not hold in this economy. 
Consider a different equilibrium concept, namely that of reproducible solution proposed by Roemer ([13], [14]). It is assumed that capitalists maximise profits, subject to the constraint that they must be able to layout the operating costs of capital in advance, whereas wages are paid out at the end of the production process. Formally, assuming stationary expectations on prices ([13]), capitalist $\nu$ 's programme is given by:

$$
\begin{aligned}
\text { choose } \alpha^{\nu} & \in P \text { to maximise } p \bar{\alpha}^{\nu}-\left(p \underline{\alpha}^{\nu}+w \alpha_{0}^{\nu}\right) \\
\text { s.t. } p \underline{\alpha}^{\nu} & \leqq p \omega^{\nu} .
\end{aligned}
$$

The set of production processes that are the optimal solutions of the above problem is denoted by $\mathcal{A}^{\nu}(p, w)$. Then:

Definition 4 ([13], [14]): $A$ reproducible solution (RS) for an economy $E \in \mathcal{E}$ is a pair $\left((p, 1),\left\{\alpha^{\nu}\right\}_{\nu \in K}\right)$, where $p \in \mathbb{R}_{+}^{n}$ and $\alpha^{\nu} \in P$ for all $\nu \in K$, such that:

(a) $\forall \nu \in K, \alpha^{\nu} \in \mathcal{A}^{\nu}(p, 1)$ (profit maximisation);

(b) $\widehat{\alpha} \geqq \alpha_{0} b$ (reproducibility),

where $\widehat{\alpha} \equiv \sum_{\nu \in K}\left(\bar{\alpha}^{\nu}-\underline{\alpha}^{\nu}\right)$ and $\alpha_{0} \equiv \sum_{\nu \in K} \alpha_{0}^{\nu}$;

(c) $\underline{\alpha} \leqq \omega$ (availability of capital), where $\underline{\alpha} \equiv \sum_{\nu \in N} \underline{\alpha}^{\nu}$ and $\omega \equiv \sum_{\nu \in K} \omega^{\nu}$;

(d) $p b=1$ (subsistence wage).

In other words, at a RS, (a) capitalists maximise profits; (b) aggregate output is sufficient to replace capital used up and for workers' consumption, and (c) aggregate capital is sufficient for production plans. Part (d) is the condition of labour market equilibrium. Note that feasibility requires that $\alpha_{0} \equiv \sum_{\nu \in K} \alpha_{0}^{\nu} \leqq \sum_{\nu \in W} l^{\nu} \leqq|W|$ (which is a standard assumption in Marxian economics). ${ }^{8}$

The next theorem proves that if Matsuo's definition of exploitation is adopted, the FMT does not hold at a RS of the economy.

Theorem 4: There is an economy $E \in \mathcal{E}$ such that at a RS the maximal profit rate is zero, while MLEU-exploitation exists.

Proof. Consider the same von Neumann production technology $(A, B, L)$ and the same bundle of wage goods $b$ as in the proof of Theorem 3. Let

\footnotetext{
[14].

${ }^{8}$ For a more detailed discussion of the notion of Reproducible Solution, see [13] and
} 
$\left(\omega^{\nu}\right)_{\nu \in K}$ be such that $\omega^{\nu}=\left[\begin{array}{l}1 \\ 1\end{array}\right]$, all $\nu \in K$, so that $\sum_{\nu \in K} \omega^{\nu}=|K|\left[\begin{array}{l}1 \\ 1\end{array}\right]$. Assume that $|K| \leq|W|$. First, it is not difficult to prove that $p=(0,1)$ is a competitive equilibrium price for this economy. In fact, it is immediate to show that the maximal profit rate is zero and if $x^{\nu} \in\left\{\left(x_{1}^{\prime}, x_{2}^{\prime}\right) \in \mathbb{R}_{+}^{2} \mid x_{1}^{\prime}+x_{2}^{\prime}=1\right\}$, then $x^{\nu} \in \mathcal{A}^{\nu}(p, 1)$, for all $\nu \in K$, with $A x^{\nu}=\omega^{\nu},(B-A) x^{\nu} \geqq b$, and $L x^{\nu}=1$, all $\nu \in K$. Therefore, noting that $|K| \leq|W|$, it follows that $\sum_{\nu \in K} L x^{\nu} \leq|W|, \sum_{\nu \in K}(B-A) x^{\nu} \geqq \sum_{\nu \in K} L x^{\nu} b$, and $\sum_{\nu \in K} A x^{\nu}=$ $\sum_{\nu \in K} \omega^{\nu}$. Finally, $p b=1$ so that Definition $4-(\mathrm{d})$ is also satisfied.

Second, because $b \in \widehat{P}\left(\alpha_{0}=1\right) \backslash S \widehat{P}\left(\alpha_{0}=1\right)$, the desired result follows from Theorem 2.

Theorems 3 and 4 raise serious doubts concerning the strongly subjectivist approach to exploitation, and its relation with the FMT. Matsuo proposed this approach precisely in order to rescue Marxian exploitation theory from Petri's [12] counterexample, which shows that although Morishima's ([10]) Generalised FMT is robust in BGEs, the FMT does not hold in general if other equilibrium notions (such as Roemer's RS) are considered: profits can be positive even though no exploitation exists in the sense of [10]. Proposition 1 shows that Petri's counterexample can be resolved if Definition 2 is adopted: for any nonnegative price vector, if profits are positive, MLEU-exploitation exists. This is not really a solution of Petri's puzzle, however, because Theorem 3 states that if Matsuo's approach is adopted, the FMT does not hold even at a BGE. From this perspective, his subjectivist approach seems to score worse than Morishima's definition, rather than solving its difficulties.

In sum, if workers are heterogeneous and consume different bundles, the WSET cannot be generalised. Further, even if one assumes workers to consume the same bundle, the strongly subjectivist approach to exploitation does not preserve - let alone generalise - the relation between exploitation and profits, and the FMT is violated, contrary to Matsuo's claims.

As a final remark, it is worth noting that, even setting aside the doubts related to Theorems 3 and 4 above, the claims concerning the generality of the results are unwarranted, even if a representative worker is assumed. Although the class of utility functions in $\mathcal{U}$ is rather large, some important cases are excluded (for instance, perfect complements, lexicographic preferences, neutral goods), and this exclusion is puzzling if the motivation of the whole exercise is to provide a general framework that avoids counterexamples to the FMT: given the restrictions on $\mathcal{U}$, it is not difficult to build new coun- 
terexamples using utility functions outside $\mathcal{U}$. For instance, if preferences are perfect complements, then the equivalence result breaks down. So, if the approach is to be defended, this is not on the grounds of its presumed generality. One would have to argue directly that it is theoretically superior.

Matsuo defends the strict monotonicity assumption against one specific critique, namely against the claim that workers may not derive welfare from accumulation goods. However, on the one hand, this does not respond to cases such as perfect complements or lexicographic preferences. On the other hand, to postulate that "workers have some preference for accumulation goods if they - even unconsciously - have some ideas about a desirable production allocation in the society" ([7], p.263) is arguably rather ad hoc and objectionable. And it implies that an approach that aims to provide a general theory of exploitation, which is robust to counterexamples, ultimately rests on a purely empirical assumption.

\section{An objectivist approach to exploitation}

The previous sections raise several doubts on Matsuo's approach, but they do not provide a complete answer to the issue of whether, and how, subjective preferences should play a role in exploitation theory. A thorough analyis of this issue goes beyond the limits of this paper, but some important points can be made, which point out some interesting lines for further research. First, the previous arguments forcefully suggest that an approach in which subjective preferences directly enter the definition of value has a number of undesirable properties. More precisely, they support the view that some minimal objectivism is necessary, whereby if all the objective features of two economies are identical, their aggregate exploitation indices should also be identical, regardless of agents' subjective preferences.

Axiom 1 (Minimal objectivism): Let $E=\left\langle K, W ;\left(u^{\nu}\right)_{\nu \in W} ; P ;\left(\omega^{\nu}\right)_{\nu \in K}\right\rangle$ and $E^{\prime}=\left\langle K^{\prime}, W^{\prime} ;\left(u^{\prime \nu}\right)_{\nu \in W^{\prime}} ; P^{\prime} ;\left(\omega^{\prime \nu}\right)_{\nu \in K^{\prime}}\right\rangle$ be such that $K=K^{\prime}, W=$ $W^{\prime}, P=P^{\prime}$, and $\left(\omega^{\nu}\right)_{\nu \in K}=\left(\omega^{\prime \nu}\right)_{\nu \in K^{\prime}}$. If $\left(b^{\nu}\right)_{\nu \in W}=\left(b^{\prime \nu}\right)_{\nu \in W^{\prime}}$, then $e\left(\left(b^{\nu}\right)_{\nu \in W}, l ;\left(u^{\nu}\right)_{\nu \in W}\right)=e\left(\left(b^{\prime \nu}\right)_{\nu \in W^{\prime}}, l ;\left(u^{\prime \nu}\right)_{\nu \in W^{\prime}}\right)$.

To be sure, there are a number of definitions satisfying Axiom 1 and the axiom is silent concerning many controversial issues in exploitation theory. For example, it may be argued that Axiom 1 should be strengthened to exclude all possible influences, direct and indirect, of subjective preferences 
and even individual choices from the analysis of exploitation. Yet, it does restrict the admissible definitions and it can be seen as the first step of a novel axiomatic analysis of preference and choice in exploitation theory.

Second, in the rest of this section an objectivist approach is considered, which provides more satisfactory answers to the two core issues analysed in this paper, namely the construction of a robust exploitation index and the validity of the FMT in general economies. Given an economy $E \in \mathcal{E}$, let $\left(\alpha,\left(b^{\nu}\right)_{\nu \in W}\right)$ be an allocation such that $\alpha \in P, \widehat{\alpha} \in \mathbb{R}_{+}^{n}$, and let $p \in \mathbb{R}_{+}^{n}$ be the associated price vector. Let $B(p, l) \equiv\left\{c \in \mathbb{R}_{+}^{n} \mid p c=l\right\}: B(p, l)$ is the set of bundles that can be afforded by workers who supply $l$ units of labour. Note $b^{\nu} \in B(p, l)$ for all $\nu \in W$. Then, consider $c \in B(p, l)$ such that $c=t \widehat{\alpha}$ for some $t>0$. Denote such $t>0$ by $t^{(p, l, \alpha)}$.

Definition 5: For any $E \in \mathcal{E}$, let $\left(\alpha,\left(b^{\nu}\right)_{\nu \in W}\right)$ be an allocation such that, $\alpha \in P, \widehat{\alpha} \in \mathbb{R}_{+}^{n}, l^{\nu}=l$, all $\nu \in W$, and let $(p, 1) \in \mathbb{R}_{+}^{n+1}$ be the associated price vector. Every worker $\nu \in W$ is exploited if and only if $l-t^{(p, l, \alpha)} \alpha_{0}>0$.

Definition 5 is conceptually related to the 'New Interpretation' ([1]; [2]; [3]) and $t^{(p, l, \alpha)} \alpha_{0}$ can be interpreted as the value of labour power. Therefore, as in the 'New Interpretation', workers are exploited if and only if the share of wages in national income is less than one. Definition 5 is superior to the subjectivist approach proposed by Matsuo in terms of providing a theoretically robust and empirically meaningful exploitation index. The definition of the aggregate index of exploitation is straightforward and no aggregation issues arise, including in general convex economies with heterogeneous agents: $e\left(\left(b^{\nu}\right)_{\nu \in W}, l,\left(u^{\nu}\right)_{\nu \in W}\right)=\frac{l-t^{(p, l, \alpha)} \alpha_{0}}{l}$. Definition 5 satisfies Axiom 1 and the latter index is well-defined and uniquely determined, for any set of objective characteristics of the economy, which allows meaningful comparisons across time and between countries concerning exploitation, and - more generally fruitful empirical analysis in a Marxian framework. ${ }^{9}$

Definition 5 is also superior in terms of preserving the classical Marxian insight concerning the relation between labour, exploitation, and profits in general convex cone economies with heterogeneous agents. In the rest of this section, it is assumed that $l=1$, without loss of generality, and $t^{(p, \alpha)}$ is used instead of $t^{(p, 1, \alpha)}$ for the sake of notational simplicity. Recall that $\beta=\sum_{\nu \in W} b^{\nu}$ and let $b=\frac{\beta}{|W|}$. Under Definition 5 , the equivalent of Theorem 2 in economies with heterogeneous agents can be proved.

\footnotetext{
${ }^{9}$ For a discussion of the empirical implications of the 'New Interpretation,' see [8].
} 
Theorem 5 (The General System of an Objectivist Exploitation Theory): For any economy $E \in \mathcal{E}$, the following statements are equivalent:

(1) $b \in \widehat{P}\left(\alpha_{0}=1\right) \backslash S \widehat{P}\left(\alpha_{0}=1\right)$;

(2) $\nexists p \in \mathbb{R}_{++}^{n}$ s.t. $p[\widehat{\alpha}-b] \leqq 0$ holds for all $\widehat{\alpha} \in S \widehat{P}\left(\alpha_{0}=1\right)$;

(3) There exists $\alpha \in P\left(\alpha_{0}=1\right)$ s.t. for all $p \in \mathbb{R}_{++}^{n}, 1-t^{(p, \alpha)} \alpha_{0}>0$.

Proof. By Theorem 2, it suffices to show $(1) \Leftrightarrow(3)$. First, suppose that (1) holds. Then, there exists $\alpha \in P\left(\alpha_{0}=1\right)$ such that $\widehat{\alpha} \geq b$, by A2 and $\mathrm{A} 3$, and for all $p \in \mathbb{R}_{++}^{n}, p \widehat{\alpha}>p b=p c$ for any $c \in B(p, 1)$. Thus, for $t^{(p, \alpha)} \widehat{\alpha} \in B(p, 1), p \widehat{\alpha}>p \cdot t^{(p, \alpha)} \widehat{\alpha}$, which implies $1-t^{(p, \alpha)} \alpha_{0}>0$, since $\alpha_{0}=1$.

Next, suppose that (3) holds. Let $\alpha \in P\left(\alpha_{0}=1\right)$ be such that for all $p \in \mathbb{R}_{++}^{n}, 1-t^{(p, \alpha)} \alpha_{0}>0$. Suppose that $\widehat{\alpha} \geq b$ does not hold. Then, there exists $p \in \mathbb{R}_{++}^{n}$ such that $p \widehat{\alpha} \leqq p b$. Thus, since $c \in B(p, 1), p \widehat{\alpha} \leqq p c$, which implies $p \widehat{\alpha} \leqq p t^{(p, \alpha)} \widehat{\alpha}$, so that $t^{(p, \alpha)} \geqq 1$. However, since $1-t^{(p, \alpha)}>0$, this is a contradiction. Thus, (3) implies that there is $\alpha \in P\left(\alpha_{0}=1\right)$ such that $\widehat{\alpha} \geq b$, which implies $b \in \widehat{P}\left(\alpha_{0}=1\right) \backslash S \widehat{P}\left(\alpha_{0}=1\right)$ by A3 and the definition of $\bar{S} \widehat{P}\left(\alpha_{0}=1\right)$.

Theorem 5 states that (3) every worker in the economy is exploited in the sense of Definition 5 (at some feasible allocation) if and only if (1) it is possible to produce $b$ with less than the one unit of labour supplied by each worker. In turn, these two conditions are equivalent to (2) the existence of strictly positive profits for any strictly positive price vector. Furthermore, it can be proved that the equivalent of Proposition 1 holds for Definition 5 and, unlike in Matsuo's subjectivist approach, the next results show that the FMT holds under standard definitions of equilibrium with $p \geq 0$.

Theorem 6 states that, under Definition 5, the FMT holds if von Neumann's equilibrium concept is adopted.

Theorem 6: For any economy $E \in \mathcal{E}$, with $P=P_{(A, B, L)}$, at any $\mathrm{BGE}$ the warranted profit rate is positive if and only if every worker is exploited in the sense of Definition 5.

Proof. Let $\pi>0$. Then, $p B x-[p A+L] x>0$. Without loss of generality, let $x$ be normalised so that $L x=1$ holds. Then, given $p b=1$, the above inequality is reduced to $p[B-A] x>p b=t^{(p, x)} p[B-A] x$ for some $t^{(p, x)}>$ 0 . Since $t^{(p, x)}<1,1-t^{(p, x)} L x=1-t^{(p, x)}>0$ holds, so that every worker is exploited in terms of Definition 5. 
Let $\pi \leqq 0$. Then, $p B x-[p A+L] x \leqq 0$. Again, given $L x=1$ and $p b=1, p[B-A] x \leqq p b=t^{(p, x)} p[B-A] \bar{x}$ for some $t^{(p, x)} \geqq 1$. Therefore $1-t^{(p, x)} L x=1-t^{(\overline{p, x})} \leqq 0$ holds, so that every worker is not exploited in terms of Definition 5 .

Theorem 6 makes Definition 5 at least equivalent to Morishima's ([10]) classical definition, from the viewpoint of preserving the FMT. Unlike the latter approach, though, under Definition 5, the Marxian postulate that exploitation is synonymous with positive profits holds even if other equilibrium concepts are adopted, such as Roemer's ([13]) RS.

Theorem 7: For any economy $E \in \mathcal{E}$, let $\left((p, 1),\left\{\alpha^{\nu}\right\}_{\nu \in K}\right)$ be a RS. Then, $p\left(\sum_{\nu \in K} \widehat{\alpha}^{\nu}\right)-\sum_{\nu \in K} \alpha_{0}^{\nu}>0$ if and only if every worker is exploited in the sense of Definition 5.

Proof. Let $\alpha \equiv \sum_{\nu \in K} \alpha^{\nu}, \alpha_{0} \equiv \sum_{\nu \in K} \alpha_{0}^{\nu}$, and $\alpha^{\prime} \equiv \frac{\alpha}{\alpha_{0}}$. Thus,

$$
p\left(\sum_{\nu \in K} \widehat{\alpha}^{\nu}\right)-\sum_{\nu \in K} \alpha_{0}^{\nu} \lesseqgtr 0 \Leftrightarrow p \widehat{\alpha}^{\prime}-1 \lesseqgtr 0 .
$$

First, suppose that $p \widehat{\alpha}^{\prime}-1>0$. Then, since $p b=1$, it follows that $p \widehat{\alpha}^{\prime}>p b=t^{\left(p, \alpha^{\prime}\right)} p \widehat{\alpha}^{\prime}$, for some $t^{\left(p, \alpha^{\prime}\right)}>0$. Because $t^{\left(p, \alpha^{\prime}\right)}<1$, it follows that $1-t^{\left(p, \alpha^{\prime}\right)} \alpha_{0}^{\prime}=1-t^{\left(p, \alpha^{\prime}\right)}>0$, so that every worker is exploited in terms of Definition 5.

Next, if $p \widehat{\alpha}^{\prime}-1=0$, then in a similar way, it can be proved that $1-$ $t^{\left(p, \alpha^{\prime}\right)} \alpha_{0}^{\prime}=1-t^{\left(p, \alpha^{\prime}\right)}=0$, so that no worker is exploited in the sense of Definition 5.

Theorems 6 and 7 arguably provide further independent support to Definition 5. If the epistemological role of the FMT is indeed as a postulate, as assumed in most of the literature, they show that Definition 5 is preferable to the main received definitions, and to Matsuo's subjectivist approach, because it allows to derive a general, robust relation between exploitation and profits in general convex economies with heterogeneous agents.

\section{Conclusions}

This paper critically analyses the subjectivist approach to exploitation proposed by Matsuo [7], in which preferences play a direct, definitional role, 
focusing on two central issues in exploitation theory, namely the definition of measures of exploitation, and the relation between labour, exploitation, and profits, in general convex cone economies. The limits of Matsuo's approach, are shown with respect to both issues and it is argued that a minimal objectivism is necessary in exploitation theory. To be sure, it is an open question whether subjective preferences should play an indirect role in exploitation theory, for instance in defining individual exploitation status as the outcome of individual leisure and consumption choices. This paper can be seen as the first step of a new axiomatic analysis of the role of preferences in exploitation theory.

An alternative objectivist approach related to the 'New Interpretation' is also briefly analysed, and it is shown that it provides theoretically robust, and empirically meaningful, indices of individual and aggregate exploitation and it preserves the FMT in general convex economies. To be sure, these findings are not sufficient to prove that the approach provides the foundations for a general theory of exploitation in advanced capitalist economies. Two shortcomings of the models analysed in this paper might be noted: the stark class structure and the neglect of agents' optimising choices. These issues are important and they represent interesting lines for further research, but some points can be made that suggest that the definition proposed in this paper may provide many interesting insights on exploitation in advanced capitalist economies. First, [19] and [21] prove that, if Definition 5 is adopted, the FMT can be extended to accumulation and subsistence economies with optimising agents and, in less polarised economies with heterogeneous endowments, it is possible to derive the full class and exploitation structure of the economy, and a robust correspondence between class and exploitation status, in equilibrium. Instead, these conclusions do not hold under the received definitions of exploitation. Second, [21] provides a complete axiomatic characterisation of Definition 5 in the context of general convex cone subsistence economies with heterogeneous optimising agents: in such context, the objectivist approach analysed in this paper surprisingly emerges as the unique definition of exploitation that satisfies a small set of rather weak axioms. ${ }^{10}$ Third, [16] shows that, if Definition 5 is adopted, the core insights of the Marxian theory of exploitation can be extended to economies with heterogeneous individual endowments, general utility functions defined over consumption and leisure, and intertemporally optimising agents.

\footnotetext{
${ }^{10}$ See [19] for an axiomatic analysis of Definition 3 in accumulating economies.
} 


\section{$7 \quad$ References}

[1] Duménil, G. (1980): De la Valeur aux Prix de Production, Economica, Paris.

[2] Duménil, G. and D. K. Foley (2008): “The Marxian Transformation Problem," in Durlauf, S.N and L.B. Blume (eds) The New Palgrave: A Dictionary of Economics, Palgrave Macmillan, Basingstoke.

[3] Foley, D. K. (1982): "The Value of Money, the Value of Labor Power, and the Marxian Transformation Problem," Review of Radical Political Economics 14, pp. 37-47.

[4] Fujimoto, T. and Y. Fujita (2008): "A Refutation of Commodity Exploitation Theorem," Metroeconomica 59, pp. 530-540.

[5] Fujimoto, T. and A. Opocher (2009): "Commodity content in a general input-output model," Metroeconomica, forthcoming.

[6] Krause, U. (1982): "Heterogeneous Labour and the Fundamental Marxian Theorem," Review of Economic Studies 48, pp. 173-178.

[7] Matsuo, T. (2008): "Profit, Surplus Product, Exploitation and Less than Maximized Utility," Metroeconomica 59, pp. 249-265.

[8] Mohun, S. (2004): "The Labour Theory of Value as Foundation for Empirical Investigations," Metroeconomica 55, pp. 65-95.

[9] Mori, K. (2008): "Maurice Potron's linear economic model: a de facto proof of 'fundamental Marxian theorem'," Metroeconomica 59, pp. 511-529.

[10] Morishima, M. (1974): "Marx in the Light of Modern Economic Theory," Econometrica 42, pp. 611-632.

[11] Nikaido, H. (1970): Introduction to Sets and Mappings in Modern Economics, North-Holland Publishing, Amsterdam.

[12] Petri, F. (1980): "Positive profits without exploitation: a note on the generalized fundamental Marxian theorem," Econometrica 48, pp. 531-533.

[13] Roemer, J. E. (1981): Analytical Foundations of Marxian Economic Theory, Cambridge University Press, Cambridge.

[14] Roemer, J. E. (1982): A General Theory of Exploitation and Class, Harvard University Press, Cambridge. 
[15] Veneziani, R. (2004): "The Temporal Single-system Interpretation of Marx's Economics: A Critical Evaluation," Metroeconomica 55, pp. 96-114.

[16] Veneziani, R. and N. Yoshihara (2008): "Globalisation and Exploitation," mimeo, Queen Mary, University of London, and The Institute of Economic Research, Hitotsubashi University.

[17] von Neumann, J. (1945): "A Model of General Economic Equilibrium," Review of Economic Studies 13, pp. 1-9.

[18] Yoshihara, N. (1998): "Wealth, Exploitation, and Labor Discipline in the Contemporary Capitalist Economy," Metroeconomica 49, pp. 23-61.

[19] Yoshihara, N. (2009): "Class and Exploitation in General Convex Cone Economies," forthcoming, Journal of Economic Behavior and Organization.

[20] Yoshihara, N.(2007a): "An Axiomatic Approach to the Fundamental Marxian Theorem", mimeo, IER, Hitotsubashi University.

[21] Yoshihara, N. and R. Veneziani (2008): "The Injustice of Exploitation. An Axiomatic Approach," mimeo, The Institute of Economic Research, Hitotsubashi University, and Queen Mary, University of London.

[22] Yoshihara, N. and R. Veneziani (2009): "Objectivist versus Subjectivist Approaches to the Marxian Theory of Exploitation," IER Discussion Paper No. 514, The Institute of Economic Research, Hitotsubashi University. 


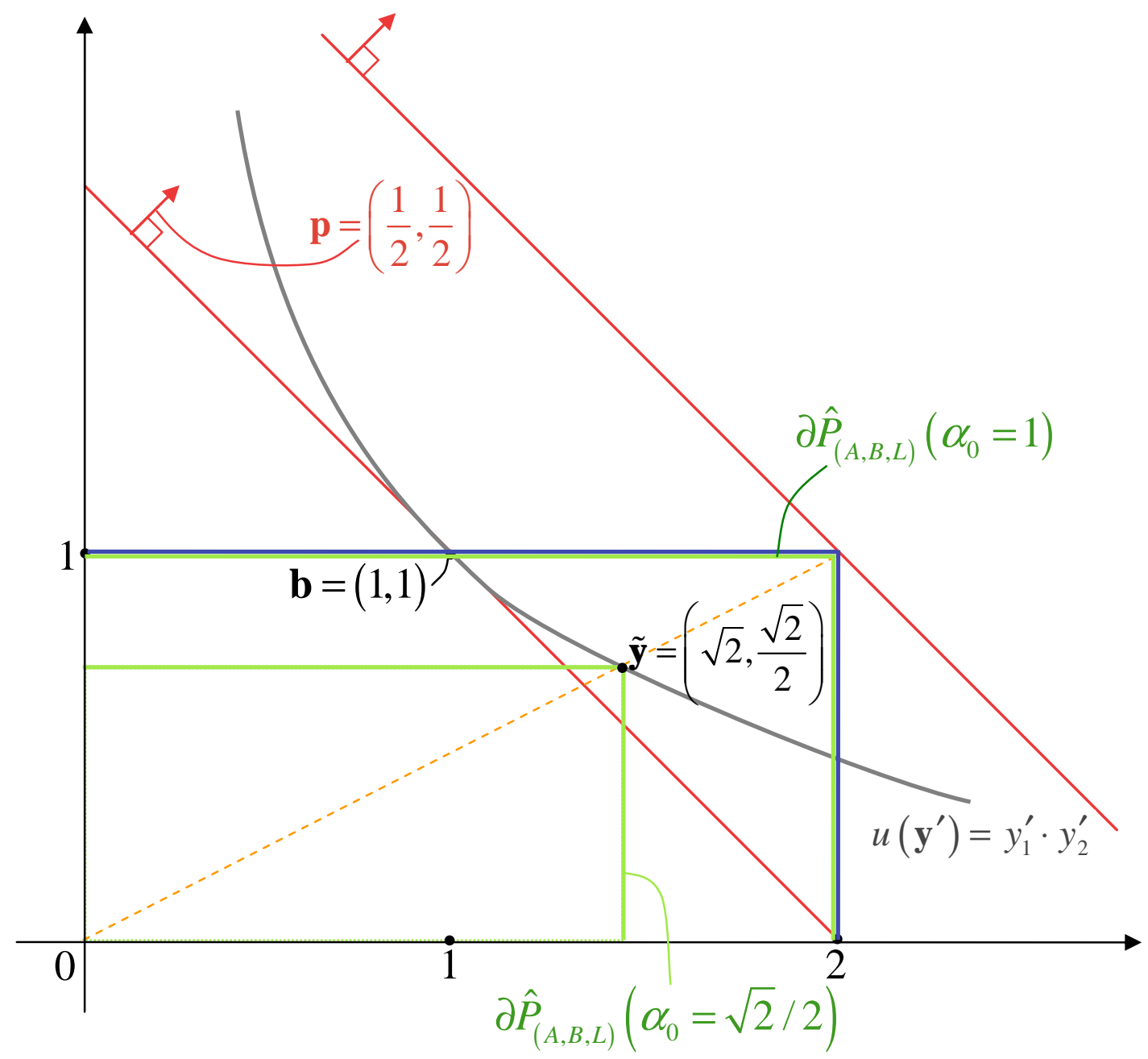

Figure 1 


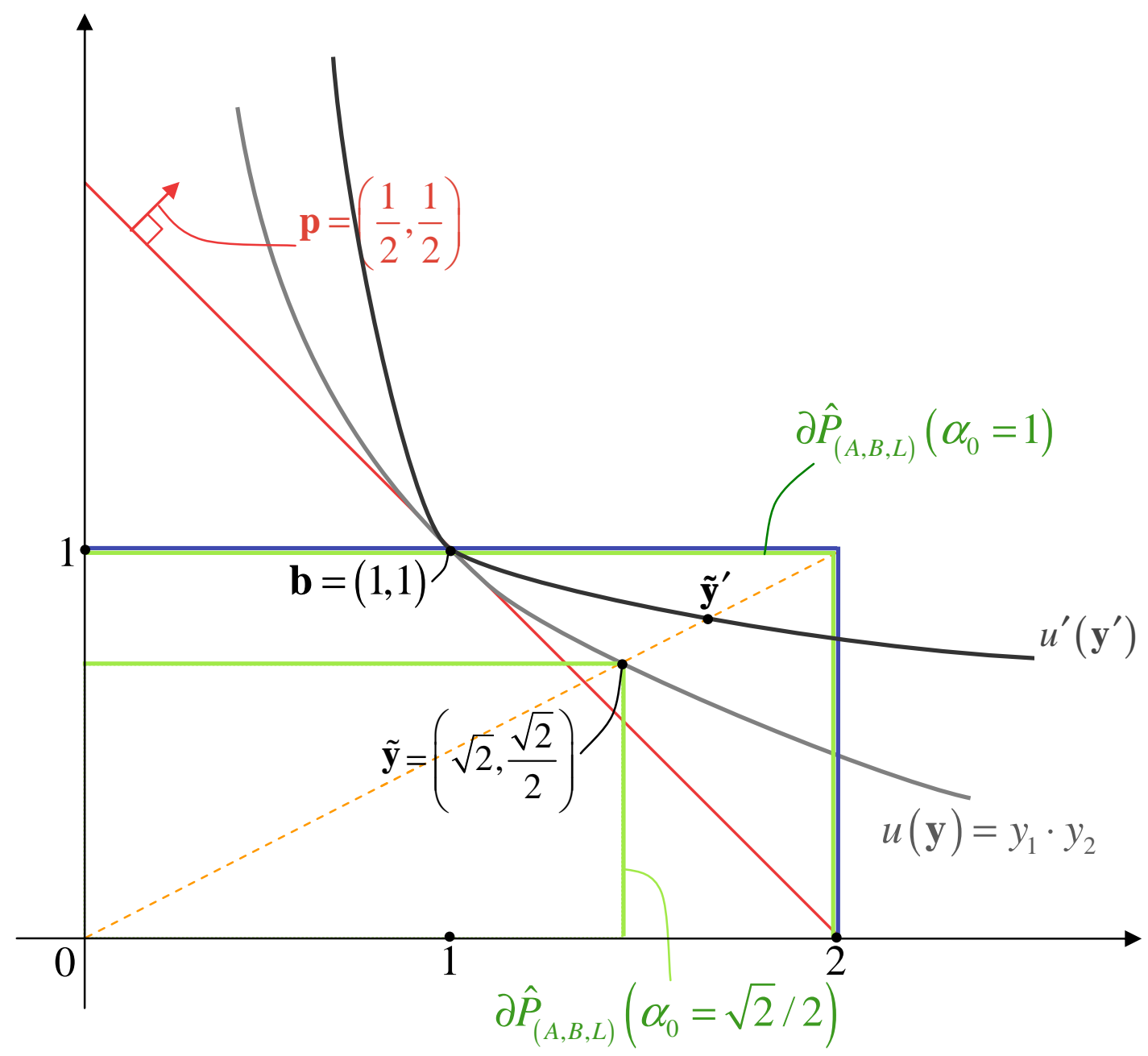

Figure 2 